MOTJGE

POATLNS OF THAS PEPAPT ARE ILLEQIBLE

It has been reproduced trom the best avallable copy to permit the broadest possible avaltability.
UCID-20264

\title{
SURVEY OF INITIAL EXPERIMENTS ON ATA BEAM DYNAMICS
}

D. S. Prono, G, J. Caporaso, Y. P. Chong, T. J. Fessenden, R. E. Hester, E. J. Lauer, T. J. Orzechowski, A. C. Paul, J. T. Weir

\section{June 25,1984}

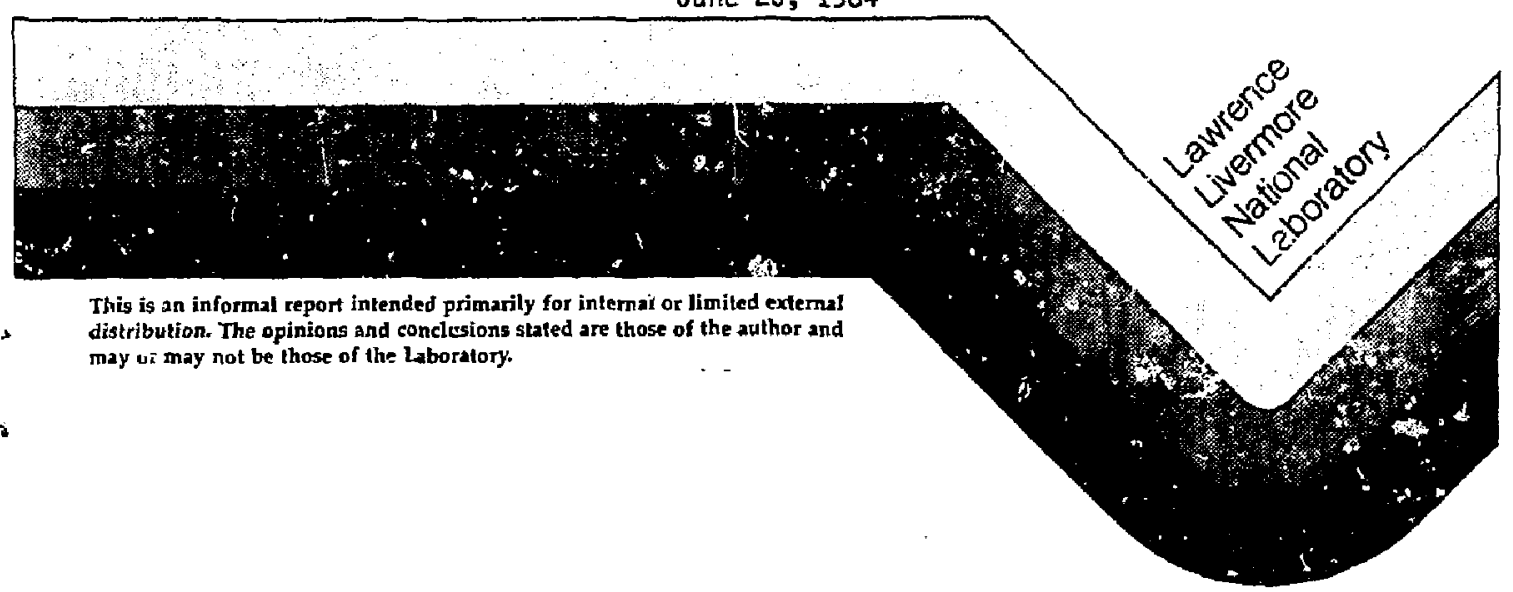

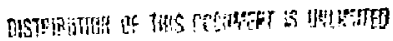




\section{SURVEY OF INITIAL EXPERIMENTS ON ATA BEAM GYNAMICS}

D. S. Prono, G. J. Caporaso, Y. P. Chong, T. J. Fessenden, R. E. Hester, E, d. Lauer, T. J. Orzechowski, A. C. Paul, J. T. Weir

Lawrence Livermore National Laboratory University of California

Livermore, California 94550

June 25,1984

UCID--20264

DE85 004410

ABSTRACT

The Advanced Test Accelerator (ATA) is a linear induction electron accelerator whose design parameters are $50 \mathrm{MeV}, 10 \mathrm{kA}$ and $70 \mathrm{~ns}$ pulse duration. The key physics issues affecting performance of the accelerator involve beam dynamics during transport through the accelerator structure. In this report, we present experimental results describing the initial operating phases of ATA. These results illustrate the complexity of the beam transport phenomena but also indicate the means to stabilize beam dynamics. Improvements in beam transport which resuit from deploying various stabilization techniques are also presented.

Hork performed jointly under the auspices of the U. S. Department of Energy by Lawrence Livermore National Laboratory under Contract $\mathrm{W}-740 \mathrm{~J}-\mathrm{ENG}-48$ and for the Department of Defense under Defense Advanced Research Projects Agency ARPA Order No. 4395 Amendment Ho. 31, monitored by Naval Surface Weapons Center under document number N60921-84-W0080. 


\section{INTRODUCTION}

The Advanced Test Accelerator is a linear induction electron accelerator which is configured as follows. The electron beam is created in the injector which nominally produces a $10 \mathrm{kA}, 2.5 \mathrm{MeV}, 70$ ns pulse. The operating performance and characteristics of this injector have been described in a previous report. ${ }^{1}$ Imediately upon being accelerated to $2.5 \mathrm{MeV}$, the beam enters a continuous solenoidal transport system. The axial magnetic field is varied over the first four meters of transport so that the beam is gradually focused down from an initial $\sim 12.5 \mathrm{~cm}$ radius to $-2 \mathrm{~cm}$ radius. At this point, the beam enters a series of accelerating cavities. A cross-section of one such accelerating cavity is shown in Figure 1. As shown in Figure 1, each accelerating cavity has its own solenoid capable of $3 \mathrm{kG}$ fields. These cavities are irduction accelerator cores which operate on the principle of an auto transformer. An input primary power pulse of $250 \mathrm{kV}$ is fed into the cavity and completes a high impedance inductive 10op. The cavity is configured such that the ful potential crop across this inductive loop diso appears across the accelerator gap. In passing through this accelerator gap, the electron beam and its image wall return current compiete a sexondary current loop which inductively couples to the primary power circuit. In this manner, the kinetic energy of the electron beam is increased by the full $250 \mathrm{kV}$ potential of the single accelerating core. In Figure 2, we indicate the equivalent circuit of an accelerating core. Note that the beam is represented as a constant current source; this implies that the time variation of the cavity's magnetic fields induced by the beam loads the cavity and alters the accelerating potential. This condition ultimately couples back to 
the beam and influences beam dynamics. Also, note that the accelerating gap of each cavity (see Figure 1) necessarily forms a discontinuity in the vacuum transport. At each such break in the circular cross-section boundary, there is no longer a balance of electric and magnetic fields (the radial electric field is slightly distorted but the azimuthal magnetic field is no longer bounded). This locolized imbalance of the beam's magnetic and electric fields a)so affects beam transport.

The first thirty accelerating cavities are assembled in six modules, each of which has five cavities alternately spaced by a solenoidal magnet. Since the beam energy in this beginiting portion of the accelerator is low, the confining axial magnetic field is held to values well below the limits set by virtual cathode formation. Since the beam breakup instability and image displacement instabilities are accentuated in transport regions of $h$ : yil gap density and low magnetic fields, the initial portion of the accelerator has the wide spacing between accelerator gaps (alternating with solenoids) in order to gradually and smoothly increase the axial field and beam energy. Beyond this point (beam energy greater than $10 \mathrm{MeV}$ ), the remaining 160 accelerator cavities are configured into 16 modules of ten cavities directly adjoining each other. Such a 10-cavity module is shown in Figure 3. For these modules, the magnetic field is usually set at a uniform nigh value of $-3 \mathrm{kG}$. This field uniformity is allowed for bean energies greater than $\sim 10 \mathrm{MeV}$ since the beam is emittance dominated. Consequent $1 \mathrm{y}$, the space change component of the beam envelope equation can be ignored and the $\gamma$ independent beam equilibrium radius is only dependent on source emittance and axial magnetic field. 
Between each 5 or 10 cavity module is a vacuum pumping station. Discrete solenoids form the section of the transport thereby making the axial magnetic field nearly continuous. This section of transport is also equipped with an $x-y$ steering coil set and a diagnostic unit. The standard diagnostic unit is a resistive current monitor (giving total current and two-dimensional centroid measurements), an array of $\dot{B}_{\theta}$-rf loops (giving a signal proportional to $\frac{d}{d t}(I / R)$ and also recording the $r f$ noise spectrum of the beam), an $x-r a y$ plunging probe (which yields a radial beam density profile by scanning a small high $Z$ target radially across the beam). These three measurements techniques are used the entire length of $A T A$ and form the core accelerator diagnostics. Additional measurements which also help characterize accelerator performance are:

i) gated TV's viewing beam intercept foils which also indicate beam profile;

ij) beam enittance and beam energy measurement techniques;

iii) determination of the magnetic profile through the entire accelerator, both axial field strength and $x-y$ steering coil strength.

\section{FIRST EXPERIMENTAL OBSERVATIONS}

Initial experiments with the ATA involved accelerating the beam through the first six accelerator modules (five accelerator cavities per module) to an energy of $10 \mathrm{MeV}$. Sample data from these initial experiments are shown in Figures $4 a$ and $4 b$. For these initial experiments, steering coils were not yet operationa1. Consequently, no corrections could be made to maintain the beam 
centroid on.axis. Data shown in Figure $4 \mathrm{a}$ show the $7 \mathrm{kA}$ beam current at entrance to the accelerator modules; the beam centroid is slightly $(\sim 3 \mathrm{~mm})$ off axis. By the $10 \mathrm{MeV}$ location, the relatively quiescent input beam current profile had become distorted by the appearance of large notches and spikes. The offset of bean centroid had grown significantly to $-1.1 \mathrm{~cm}$ accompanied by large spatial oscillations at the beginning and end of the beam current pulse (time of maximum $d I / d t$ ). Monitors of $x-$ ray emission from the accelerator wall showed spikes of current intercepting the accelerator vacuum wall. The $\AA_{\theta}$-probes located along the accelerator show a progressive increase in beam offset during the time of high $\vec{B}_{\theta}$ with a characteristic frequency increasing the further the beam is transported. Also apparent in the most downstream $r f$ loop is the $-800 \mathrm{MHz}$ oscillation characteristic of the beam breakup (BBU) cavity-interaction mode. Since the $\vec{B}_{\theta}$ probe senses $\frac{d}{d t}(I / R)$, the indicated amplitude of the spatial oscillations is weighted by the oscillation frequency; thus the lower frequency motion of the beam head and tail is far more severe than the BBU oscillations.

The lower frequency disruptions of the beam pulse clearly represent unwanted beam dynamics adversely affecting beam transport. Minimizing these oscillations occurs when the beam rise time is sharply decreased. Data shown in Figure 5a show that a current pulse with a times 6 slower rise time (dI/dt $\sim 1.3 \times 10^{11} \mathrm{~A} / \mathrm{sec}$, compared to previous $\mathrm{dI} / \mathrm{dt} \sim 8 \times 10^{11} \mathrm{~A} / \mathrm{sec}$ ) is accelerated to $10 \mathrm{MeV}$ with minimum pulse disruption. However, the end of the beam putse still has a high JI/dt and pulse disruption is apparent on the oscilloscope traces showing beam current, beam centroid, and $\dot{B}_{\theta}$. We note that the slow bean still develops centroid offset (again, no compensating 
steering coils were used). Although the beginning of the beam is relatively centered on axis but that the end of the beam pulse, immediately preceding the fast $i$ turn-off, is offset. As previously mentioned, the beam current inductively loads the accelerating cavities during the pulse rise and fall time. Shown in Figure $5 b$ are traces of an accelerator cavity voltage when the beam current has a fast and slow rate of rise. Loading due to the fast $\vec{I}$ clearly induces $a \dot{V}$ on the time changing beam.

Explanation of these initial observations inciudes two phenomena. First, at low energy, the beam axial magnetic transport system is dominated by the balance of magnetic field and space charge forces. If the magnetic field is set to have the peak current in radial equilibrium, then inevitably the current rise and fall is "mismatched" resulting in radial oscillations. Figure 6 shows computations of the beam envelope new match for a $10 \mathrm{kA}$, 2.5 MeV beam leaving the ATA injector, being transported through and accelerated by the first two five-cavity modules. The z-axial magnetic field increases from $200 \mathrm{G}$ to $2 \mathrm{kG}$ and the beam gains $250 \mathrm{keV}$ at each designated accelerator gap. The $10 \mathrm{kA}$ beam has a relatively smooth envelope indicating that the magnetic field variation is well "matched." However, the lower current values indicate that the beam beginning and end is "mismatched" and radial oscillations of the beam envelope result. This phenomenon may partially explain some of the current loss (wall x-ray signais) and degradation of the rising and falling portions of the transported bean current.

The second mechanism affecting beam motion is referred to as beam corkscrew dynamics ${ }^{2}$. This phenomenon simply involves the normal helical motion of an offset beam propagating off axis in an axial magnetic field, with the added complication of there being a slight sinusoidal energy variation. 
For a bean with initial conditions at $z=0$ of $x=2 x_{0}, \frac{d x}{d z}=k x_{0}$, and $Y=\frac{d y}{d z}=0$, the equations of motion are simply sinusoidal in $k Z$. However, if there is a temporal energy variation, (i.e., $\left.Y=\gamma_{0}\left(1+\frac{\Delta Y}{\gamma_{0}} \sin \omega \tau\right)\right)$, then the equations of motion are a sumation of harmonics in w. The amplitude of the Nth harmonic is approximately $2 x_{0}\left(\frac{K_{0} Z}{2} \frac{\Delta \gamma}{\gamma}\right)^{n}$, so that the uniforn offset equal to $2 x_{0}$ becomes a time-varying displacement of amplitude growing asymptoticaliy to a value of $2 \mathrm{x}_{0}$ and with the frequency upshifting for greater transport lengths $z$. This model explains why the fast $i$ bean with the sinusoidal-like loading of the accelerator cavity voltage has a disruptive beam transport characteristic. The frequency upshift is clearly apparent in the data and closer examination of selected traces of $x-y$ beam centroid motion does indicate (see figure 7) beam helical precession.

In subsequerit experiments with beam acceleration and transport through to $10 \mathrm{MeV}$, deployment of steering coils gave us the capability of maintaining beam centroid on axis and of developing greater finesse in tuning the accelerator-both in terms of the setting of the axial magnetic field for better matching and also in injector operational characteristics. Figure 8 shows measurements of beam current and centroid movement at the injeccor output and at the $10 \mathrm{MeV}$ accelerator location. There is obvious improvement in performance, but the disruptive beam dynamics have only been reduced, not eliminated. Ouring these experiments, we observed that relatively subtle changes in injector operation did affect beam dynamics. The waveforms shown in Figure 9 indicate the sensitivity of the total transmitted current at the $10 \mathrm{MeV}$ location to settings of the injector's bucking coil ${ }^{1}$ and the cathode jgnitor timing. The changes in injector operation did not produce any 
observable change in macroscopic beam characteristics $(1, \hat{x}, \hat{y})$ as the beam emerged from the injector. However, after beam transport through six five-cell accelerator modules, these same macroscopic observables showed the appearance of disruptive beam dynamics that were controllable by injector operating conditions. These subjective observations indicate that microstructure of the injector output beam contributes to transverse centroid fluctuations.

Data from $x$-ray plunging probes determine the radial profile of the beam current density within the accelerator. Such data are shown in Figure 10. These data indicate a temporal variation of the radial distribution of beam density during the beam pulse as well as asymmetric wings to the bean profile. Although the beam density in the wings is low winen weighted by $R^{2}$, the total amount of current carried in the asymmetric wings is not small (up to $-30 \%$ of the total beam current). The presence of these wings indicates that the injector beam output nas a class of particles whose orbits undergo large radial excursions.

Recognizing the asymetry of the wings in the beam profile, we deployed a collimator between the injector and first accelerator module. This collimator was $4 \mathrm{~cm}$ diameter and was 1.06 meters long. The axial magnetic field through the collimator was held at $\sim 1 \mathrm{kG}$. This collimator obviously bounded phase space of the beam (i.e., was an emittance filter). The beam that passed through the collimator entered the accelerator with its centroid well behaved $\left(x=y=\frac{d x}{d z}=\frac{d y}{d z}=0\right)$. The maximum "filtered current through the coll imator always was 3 to $3.5 \mathrm{kA}$ independent of the injecior output current which varied from 4 to $8 \mathrm{kA}$. The filtered current pulse was sufficiently well behaved such that it could be accelerated to $20 \mathrm{MeV}$ (through six five-cell accelerator 
modules and four ten-cell modules). The data of Figure 11 show how the collimator limits transmitted current and sharpens pulse rise time (eclipses the mismatched low-current which undergo radial pulsations). The remaining data indicate that total current, centroid motion, and $\dot{B}_{0}$ of the beam at $20 \mathrm{MeV}$ are minimally distorted by the low frequency, disruptive beam dynamics.

The collimated beam experiments suggest that the injector's output beam has a class of particles capable of executing large radjal excursions into regions of bad (highly scalloped) magnetic field. The loss of such particles to the vacum wall would cause abrupt centroid displacements. Eliminating these particles before they eneter the accelerator structure and ensuring that the beam is properly directed $\left(x=y=\frac{d x}{d z}=\frac{d y}{d z}=0\right)$ then ailows the beam centroid to be maintained on axis, thereby limiting the onset of transverse motion that leads to disruptive beam dynamics. With a collimated beam, the dynamics of the beam transport through the entire accelerator was studied. Figure 12 shows samples of beam current, centroid and $B_{\theta}$ characteristics of the beam at the end of the accelerator. For these conditions, the collimated beam was accelerated and transported through all cavities. One group of data shows beam characteristics resulting from using the $4 \mathrm{~cm}$ diameter collimator. "Corkscrewing" at the beam rise and fall are still apparent but the central high current portion of the beam body is stable. Also, for this condition there is no current loss through the accelerator. The second data group resulted from using a $6 \mathrm{~cm}$ diameter collimator. For this larger collinator, the degradation in beain dynamics throughout the entire pulse is clearly apparent with a current loss distributed throughout the accelerator. This behavior further suggests that a fraction of the total injector output pulse undergoes poor transport optics. 
With a collizated beam, more controlled experiments could be performed. The aim of these experiments was to investigate other possible causes of unwanted beam motion and current loss. Our conclusions from these are as follows:

i) that beam dynamics were not caused by pulse power drive asymmetries;

ii) that the bean dynamics were not : -used by collective effects (current dependence was weak if at ali important); and that transverse fieid errors of the accelerator solenoids were small and within tolerance. However, even small transverse field errors can cause offsets of the beam centroid. Consequently, we are minimizing all possible sources of field asymetries.

During the course of these experiments, we found why the steering coils exhibited far weaker control of the beam than anticipated. These steering coils were located over the current monitors which housed an annular ring of ferrite that effectively shorted out approximately $40 \%$ of the steering coils transverse field. Correcting this situation requires moving the steering coils to locations free of ferrite material.

A final experimental arrangement was used to further investigate the sources of transverse beam dynamics. With no collimator between the injector and the accelerator, a "wire-zone" was installed in place of the third five-cell module. Immediately beyond the wire zone was a $5 \mathrm{~cm}$ thick variable beam attenuator which had open-spaced transparencies ranging from $10 \%$ to $100 \%$. The detailed operation of a wire zone has been reported elsewhere. ${ }^{3}$ A beam exiting a wire zone, just as bean exiting a collimator, has its centroid characteristics being $x=y=\frac{d x}{d z}=\frac{d y}{d z}=0$. However, a wire zone centers the beam without' a loss of current and without bounding phase 
space (no limitation on emittance or particles capable of far off-axis orbits). These experiments, sumarized in Figure 13, indicate that after the wire zone, and regardless of the magnitude of beam current set by the variable attenuator, there is distributed current loss through the remainder of the accelerator even though the centroid motion is less than one centimeter. The results again indicate the presence of a class of beam particles from the injector that are capable of large radial orbits. The wire zone "passes" these particles but the collimator stops then. Bean transverse fluctuations on the head and tail of the pulse still occur consistent with the corkscrew mode. Development of transverse displacement within the central portion of the beam pulse is either caused by small transverse field errors or by as ymmietric current loss which causes transverse centroid motion.

\section{SUMMARY AND CONCLUSIONS}

Initial operation of ATA has demonstrated the integrity of the accelerator and its pulsed power system. Low frequency transverse fluctuations in the bean centroid have restricted the bean current to approximately $7 \mathrm{kA}$. These dynamics result from small centroid offsets being frequency upshifted due to a slight energy variation in the beam and the effect of that variation cis the normal helical motion within a solenoidal field. To avoid these deleterious bean dynamics requires continual centering of the centroid (the energy variation can be corrected but the expense of necessary power supply modifications is prohibitive). The centroid displacements are not driven by pulsed power asymmetries and do not appear to be a collective (current dependent) phenomenon. The influence of image 
displacement effects (beam centroid passing through a gap off-axis thereby having an imbalance of magnetic and electric images) has been observed, but the resultant period of these perturbations is very long and correctable by proper use of the steering coils. However, the localized loss of large orbiting beam particles that populate the asymetric wings of the beam profile causes discrete shifts in the centroid which can initiate degradation in beam transport. Using a collimator which absorbs the particles capable of large radial orbits clearly minimizes transverse fluctuations of the beam centroid and results in improved beam transport, Research is presently in progress to make a high current beam injector which does not produce particles capable of the large radial excursions. Our approach is to use high field gradient field enission cathodes with a of small area (high current density) and without a control gric. Such an injector-cathode arrangement would avoid both the marginally dense plasma that is characteristic of the present plasma surface discharge cathode and plasma-grid interaction which creates an unwanted current source. ${ }^{-1}$. Both of these phenomena may contribute to the production of bean particles that exhibit high effective emittance and large radial orbits. 
1. J. S. Prono, D. L. Birx, R. J. Briggs, Y. P. Chong, T. J. Fessenden, R. E. Hester, E. J. Lauer, T. $J_{\text {. }}$ Orzechowsk $i$, and $K$. W. Struve, First Studies of ATA Injector, August 1, 1983, Lawrence Livermore Natjonal Laboratory, UCID-19862.

2. G. L. Caporaso, et al., Beam Dynamics in the Advanced Test Accelerator (ATA), September 28, 1983, Lawrence Liverimore Nationat Laboratory, UCKL-89881.

3. D. S. Prono, et al. Electron Beam Guiding and Phase Mix Damping by an Electrostatically Charged Wire, Physical keview Letters, Volume 51, Number 9, 29 August 1983, p. 723. 


\section{F IGURE CAPIIONS}

Fig. 1. Cross section of a single accelerator cayity. Shown are the a) power input feed, b) ferrite which increases the load impedance of the shorted turn inductive lap and adds volt-seconds, c) the vacuum $250 \mathrm{kV}$ accelerator gap, d) the oil-to-vacuum insulator, e) corner reflector and mode damping ferrite which minimize higher order cavity modes, f) solenoid.

Fig. 2. Equivalent circuit of pulsed power drive and accelerator core. The electron beam load is a constant current source, the input power source from a blumlein feeding a transmission line is modeled as a constant impedance voltage source and the very high impedance cavity inductance which is resistively shunted to match to the transmission line is simply modeled as the resistive load.

Fig. 3. Shown is an assembled 10-cavity module; the cut-away section exposes the accelerating cavity and the encased solenoid.

Fig. 4a. Shown are oscilloscope traces of beam current and beam centroid position in the horizontal and vertical plane. Centroid location is given by the relation $x(t), y(t)=V_{x, y}(t) \times R / 2 V_{I}(t)$ where $R=$ vacuum pipe radius $6.7 \mathrm{~cm}, V_{I}(t)$ is the time dependent total current voltage signal, and $V_{x, y}(t)$ is the time dependent centroid voltage signal. The two sets of data show the input beam conditions and exit beam conditions after acceleration by and transport through the first thirty accelerator cavities. The $x$-ray monitoring photomultiplier shows bursts of $x$-rays caused by spikes of beam current intercepting the vacuum wall.

Fig. 4b. Shown are oscilloscope traces of the bean's $B_{\theta}$ signal $\left(=\frac{d}{d t} I / R\right)$ at various locations progressively along the accelerator. Note the signature of the corkscrew mode on the beam head (increasing frequency with propagation distance) and the eventual appearance of the $800 \mathrm{MHz}$ BBU oscillations. 
Fig. 5a. Oscilloscope traces of accelerator performance through $10 \mathrm{MeV}$ showing total current and beam centroid motion for operating conditions of a much slower bean current rise time. Note that beam centroid shows offset but no axis-crossing beam sweep.

Fig. 5b. Data showing how varying the bean current rise time affects the accelerator cavity voltage.

Fig. 6. Computations of the beam envelope equation for the first 8 meters of transport using various values of injector output beam current being transported through (while being accelerated) a realistic axial magnetic field that is matched (causing minimum radial pulsations) for high currents. As shown, lower currents (durirg beam rise and fall) are mismatched and experienre serious radial oscillations of the beam envelope.

Fig. 7. Oscilloscope traces showing details of beam centroid motion in the $x$ and $y$ planes for the rising current portion of a beam pulse. The phasing of the transverse motion in the orthogonal planes is consistent with helical beam motion.

Fig. 8. Data of beam characteristics showing beam current and $x-y$ centroid motion at the beginning of the accelerator and at the $10 \mathrm{HeV}$ location. The improved performance resulted from using steering coils to maintain beam centroid on axis and from improved operation of the injector.

Fig. 9. For slight adjustments in injector operations (as shown: top-bucking coil on/off and botton-ignitor timing $80 \mathrm{~ns} / 60 \mathrm{~ns}$ ) there is significant change in beam properties by the $10 \mathrm{MeV}$ location. For these different operating conditions, there is no discernible change in injector output beam macroscopic observables $I(t), \hat{x}(t)$ and $\hat{y}(t)$; this suggests that beam micro structure is influential in beam dynamics.

Fig. 10. Data showing radial dependence of plunging $x$-ray probe signal which is proportional to the current density. The time varying asymetric wings indicate that the injector output has a time-dependent class of particles that is capable of large radial motion. 
Fig. 11. Data of beam characteristics (beam current, $I(t)$; bean centroid motion, $\hat{x}(t), \hat{y}(t)$; and $\hat{B}_{\theta}$ signal of the beam) at the injector output, the coll imator output, and after bean iransport and acceleration to $20 \mathrm{MeV}$. The collimator, which limits current by acting as an emittance filter, transmits a portion of the beam pulse which only minimally develops transverse motion of the beam centroid.

Fig. 12. Beam characteristic's ( $I, \bar{x}, \bar{y}$, and $\bar{B}_{\theta}$ ) for beams transported and accelerated through the entire accelerator to full energy. Two sizes of collimators were used, the norma! $4 \mathrm{~cm}$ diameter and then a $6 \mathrm{~cm}$ diameter collimator. The smaller collimator injected $2 \mathrm{kA}$ into the accelerator with no subsequent current loss; the larger collimator injected $3.5 \mathrm{kA}$ into the accelerator and there resulted an $\sim 1 \mathrm{kA}$ distributed current loss throughout the accelerator and reappearance of substantial transverse centroid motion. Both experiments were conducted with identical injector configurations $13 \mathrm{~cm}$ cathode-grid gap, grip masked to $250 \mathrm{~cm}^{2}$ extraction area and using a $50 \%$ transmissive grid mesh).

Fig. 13. With a "wire zone" installed at the 5 MeV location, the beam is injected into the remaining accelerator structure both on center and forward directed. For a range of current values as prescribed by the variable attenuator, the beam centroid is seen to remain within $1 \mathrm{~cm}$ of axis, but a distributed current loss still occurs. The graphs show the results of one experiment conducted with a $6-1 / 2 \mathrm{kA}$ beam current. For all graphs, the horizontal axis represents the number of accelerator cores through which the beam propagated (i.e., z-distance, 200 cores is equivalent to 80 meters accelerator length). Top: peak centroid $x$-offset, $\mathrm{cm}$; Middle: peak centroid y-offset, cm; Bottom: beam current, kA. 
Fig. I.

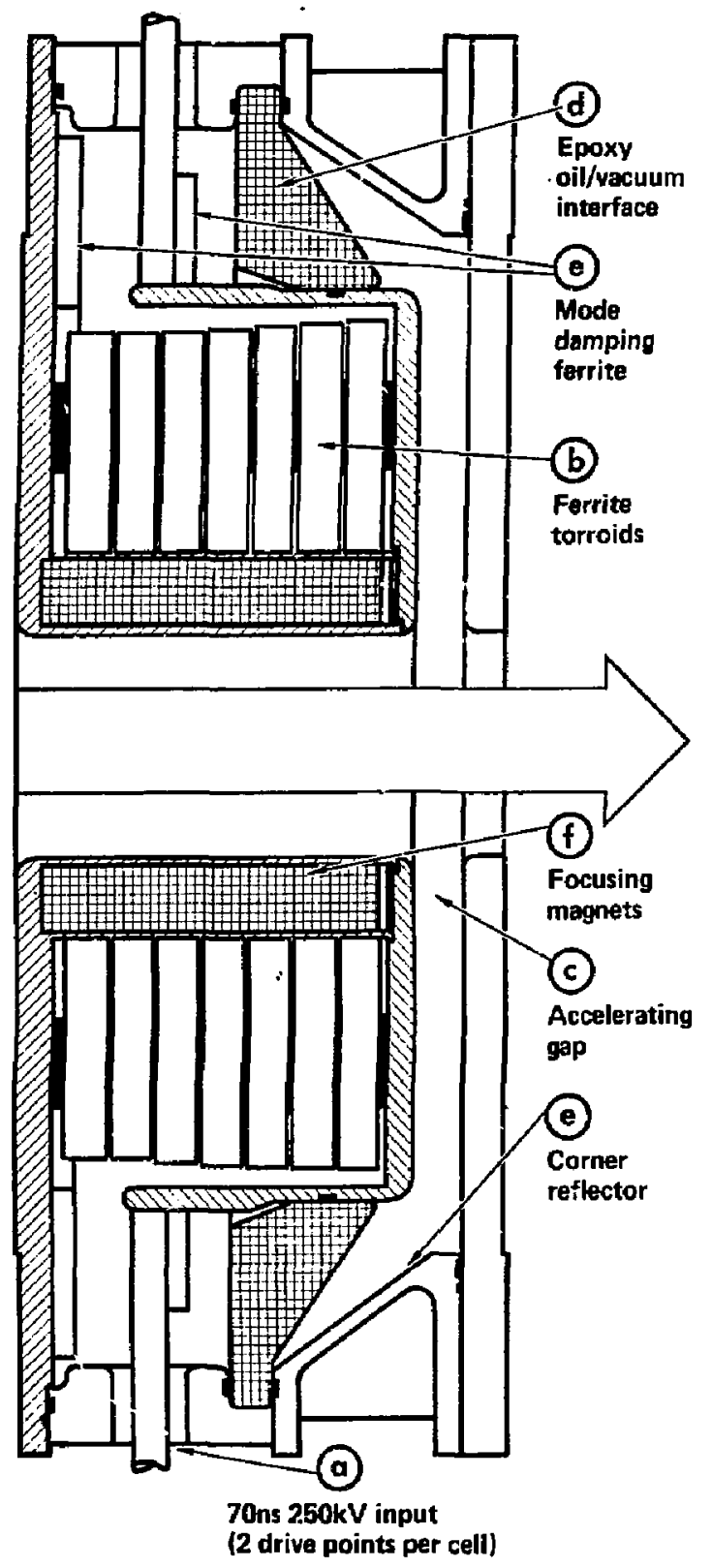

$-16=$ 
Fig. 2.

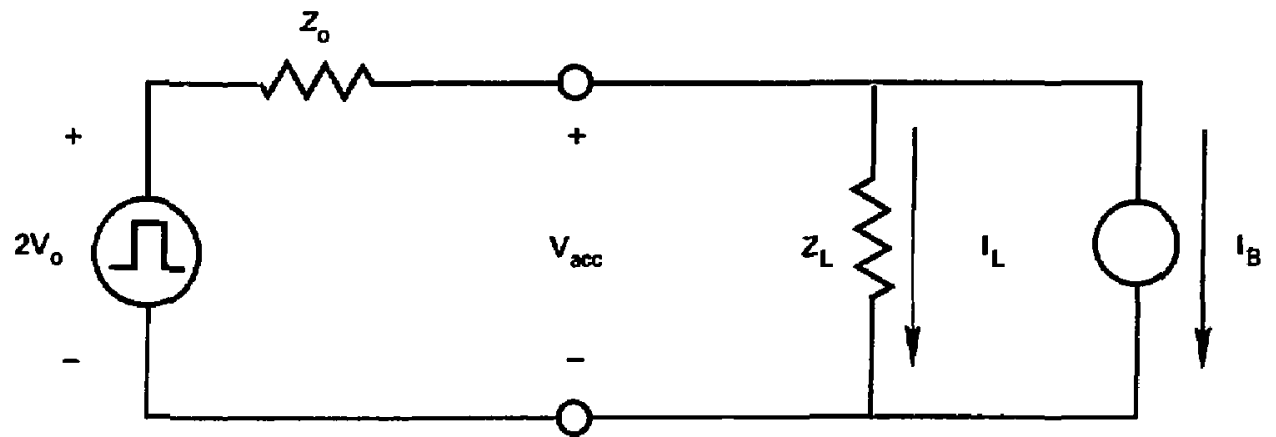

$\mathrm{v}_{\mathrm{o}}=250 \mathrm{kV}$

Note: beam is a constant

$z_{0}=8.3 \Omega$

Nominal design point : $\quad V_{a c c}=250 \mathrm{kV}$

$$
\begin{aligned}
& I_{B}=10 \mathrm{kA} \\
& I_{L}=20 \mathrm{kA}, Z_{\mathrm{L}}=12.5 \Omega
\end{aligned}
$$


Fig. 3 .

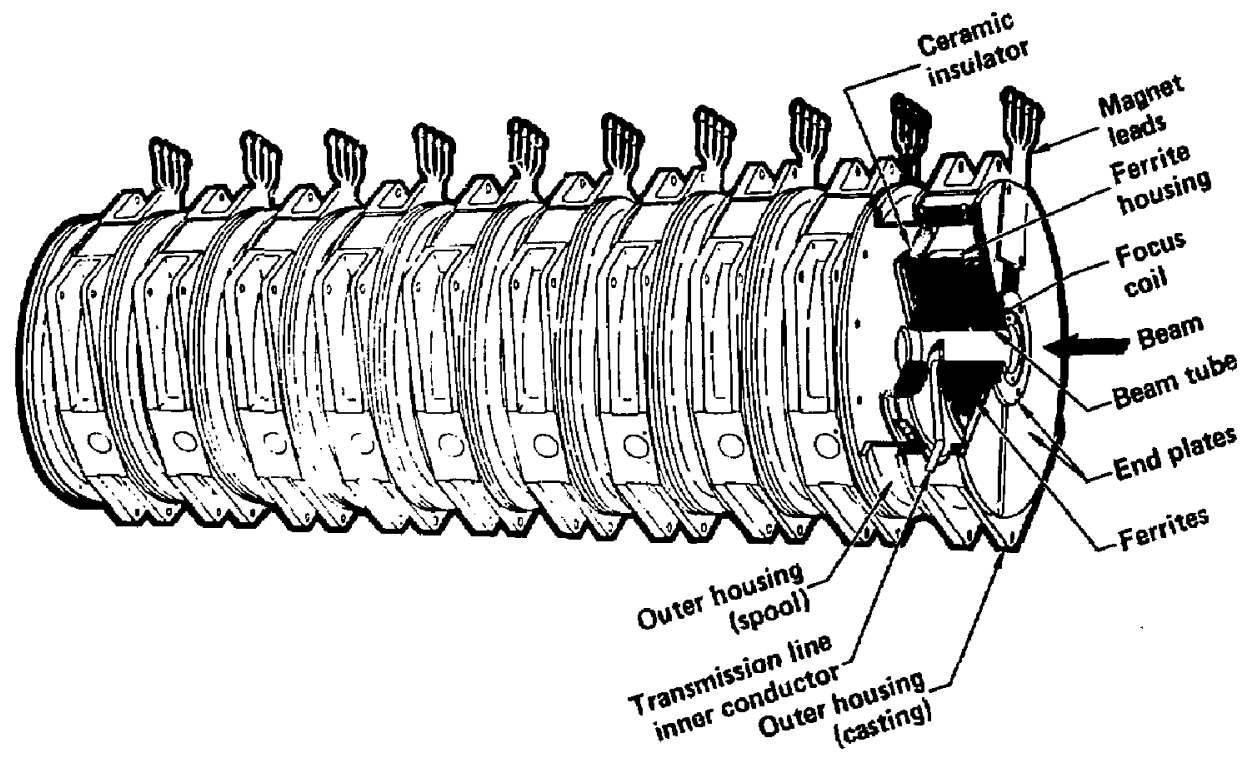


Beam Characteristics

$1 \mathrm{KA} / \mathrm{div}=100 \mathrm{mv} / \mathrm{div}$ $10 \mathrm{~ns} / \mathrm{div}$

$100 \mathrm{mv} / \mathrm{div}$

$10 \mathrm{~ns} / \mathrm{div}$

$X$ (top) $Y$ (bottam) displacement.

Injected into accelerator
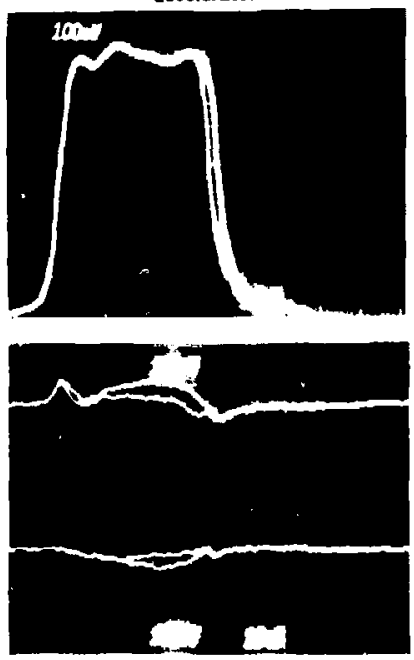

After $10 \mathrm{MeV}$ acceleration (40 cores)
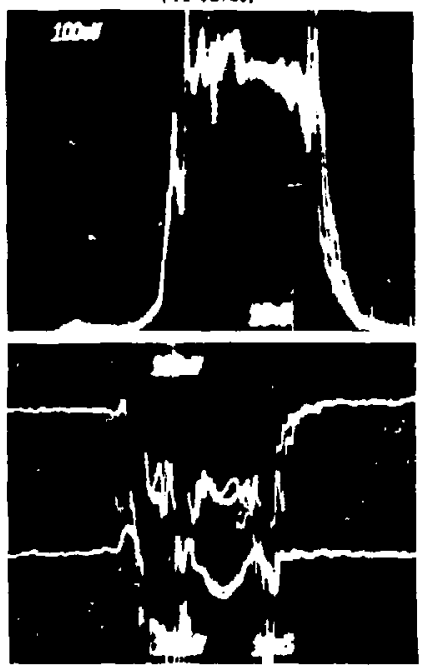

Fig. $4 a$.

$X$-ray signal emanating from vacuum wall indicating time dependent beam spilling onto wall (relative units, $10 \mathrm{~ns} / \mathrm{div}$ )
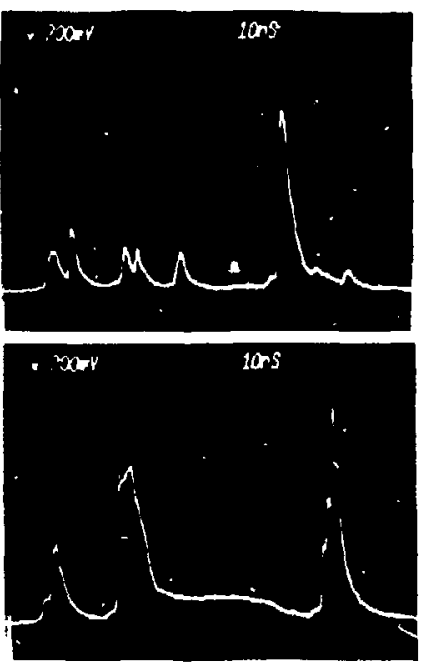
Fig. 4b.
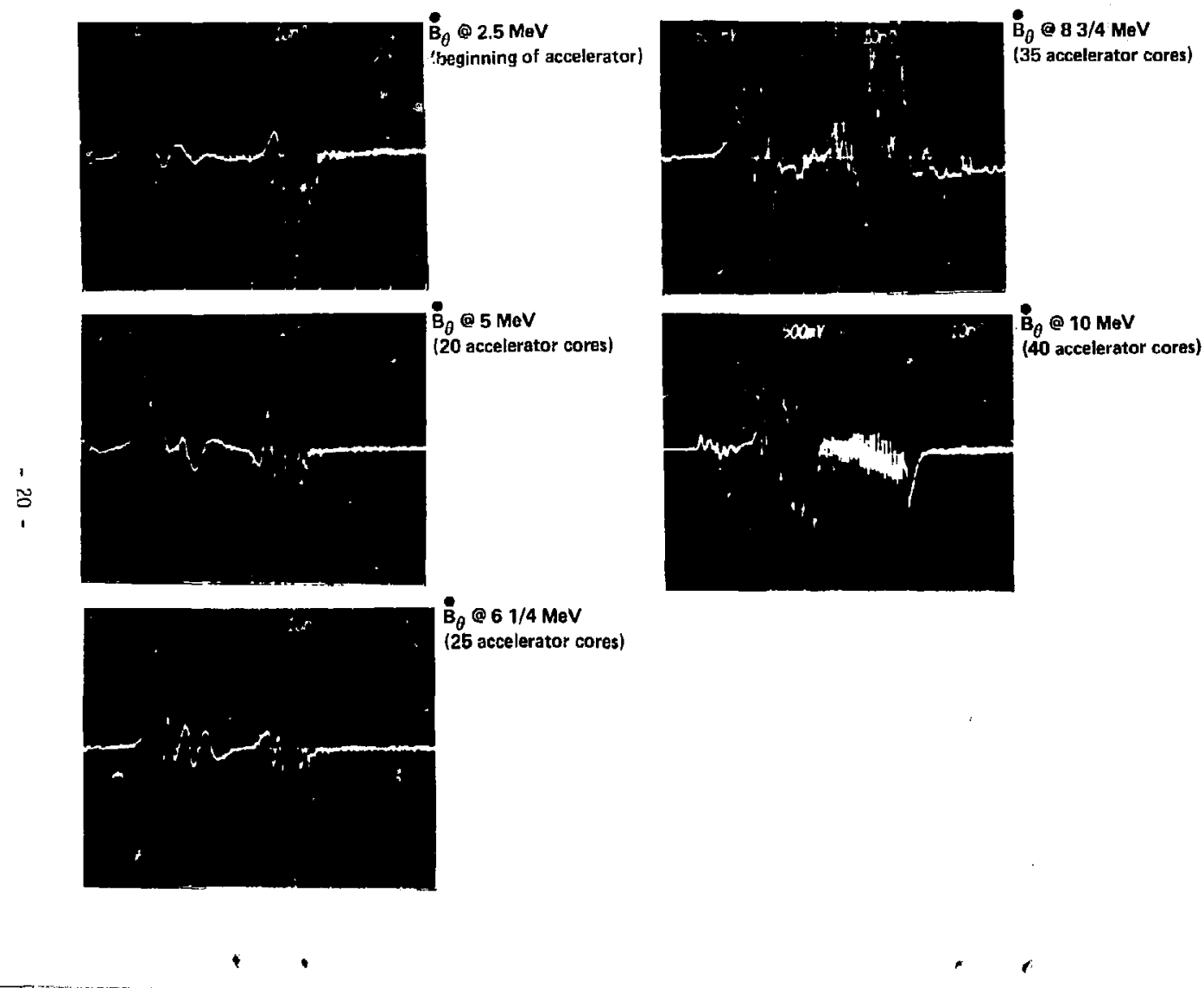

В 8 8 $3 / 4 \mathrm{MeV}$

( 35 accelerator cores)

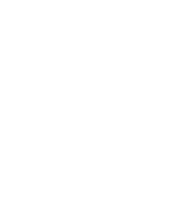

$B_{\theta} @ 10 \mathrm{MeV}$

(20 accelerator cores)

$\mathrm{B}_{\theta} @ 61 / 4 \mathrm{MeV}$

( 25 accelerator cores) 
Propagation Characteristics of Slow Rise-Time Beams

At accelerator injection

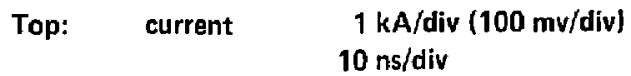

Bottom: Centroid displacement $\quad 100 \mathrm{mv} / \mathrm{div}$

$X$ (top), $Y$ (bottom) $10 \mathrm{~ns}$ div

Same scales
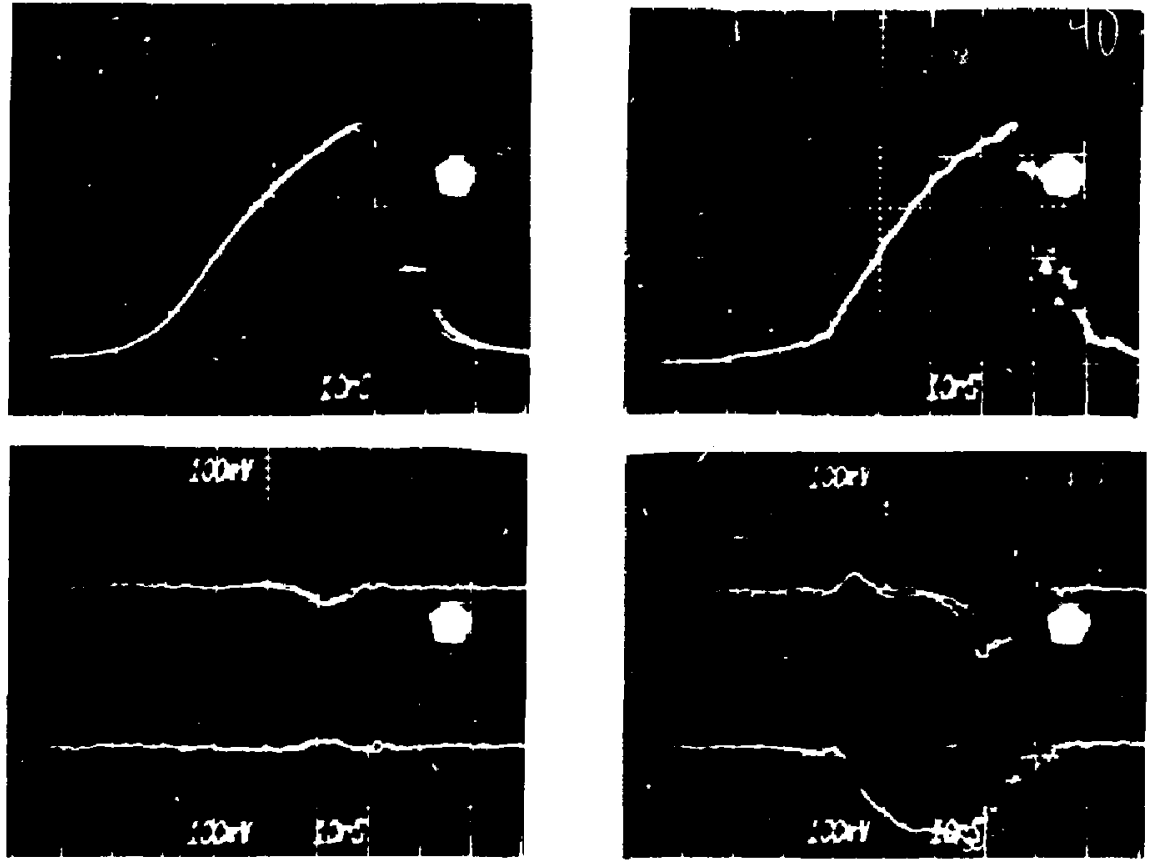
Fig. 5b.

Accelerator core voltage with fast current rise time (like Fig. 4)

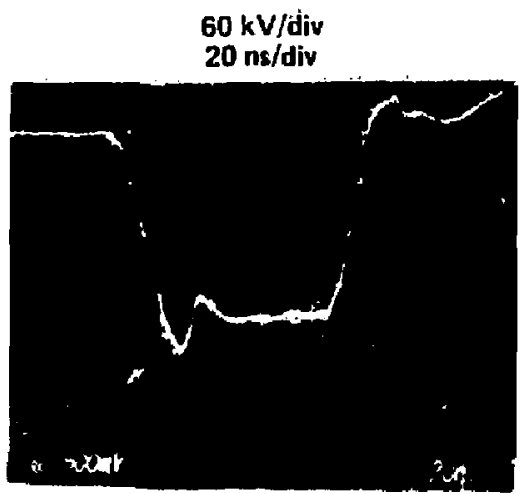

Accelerator core voltage with slow current rise time (like Fig. 5)

$60 \mathrm{kV} / \mathrm{div}$

$20 \mathrm{~ns} / \mathrm{div}$

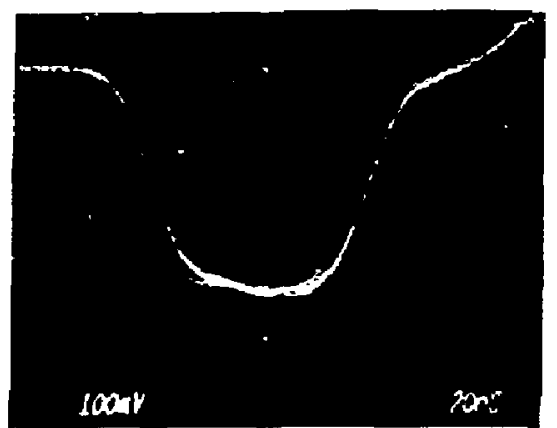


Fig. 6.

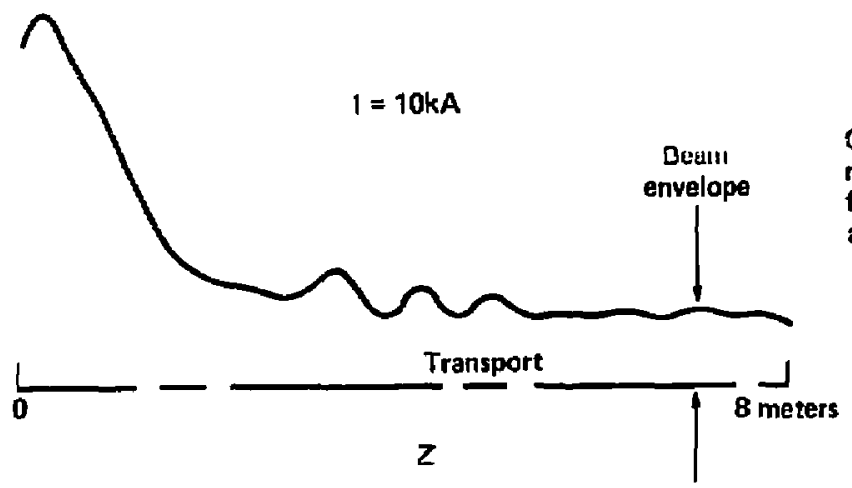

Computed beam envelope for realistic $B_{Z}(Z)$ matched (tuned) for minimum oscillators of a 10kA boam current

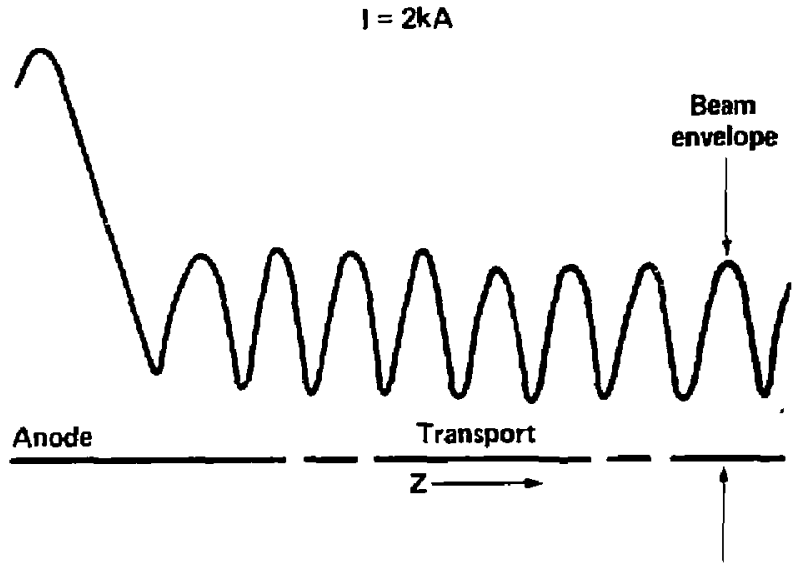

Computed beam envelope for same $B(Z)$ but $2 k A$ beam current (i.e. during current rise time) 
Fig. 7.

Top $X$ centroid motion

Bottom $Y$ centroid motion

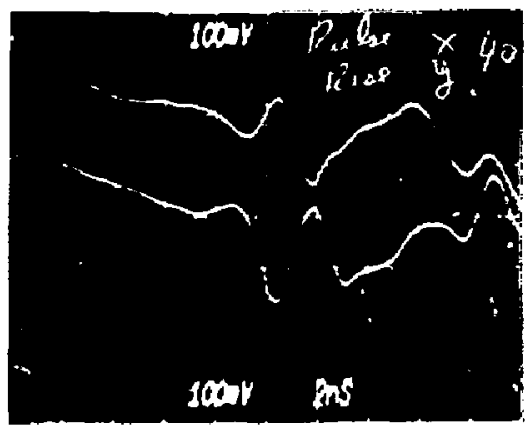


Fig. 8.

Beam Characteristics

At accelerator input

Top photo: current $2 \mathrm{kA} / \mathrm{div}$ (200mv/div) $10 \mathrm{~ns} / \mathrm{diy}$

$\begin{array}{llr}\text { Bottom } & \text { Beam centroid } & 200 \mathrm{mv} / \mathrm{div} \\ \text { pholo: } & \text { displacement } & 10 \mathrm{~ns} / \mathrm{div}\end{array}$

Top $X$ displacement

Bottom $Y$ displacement
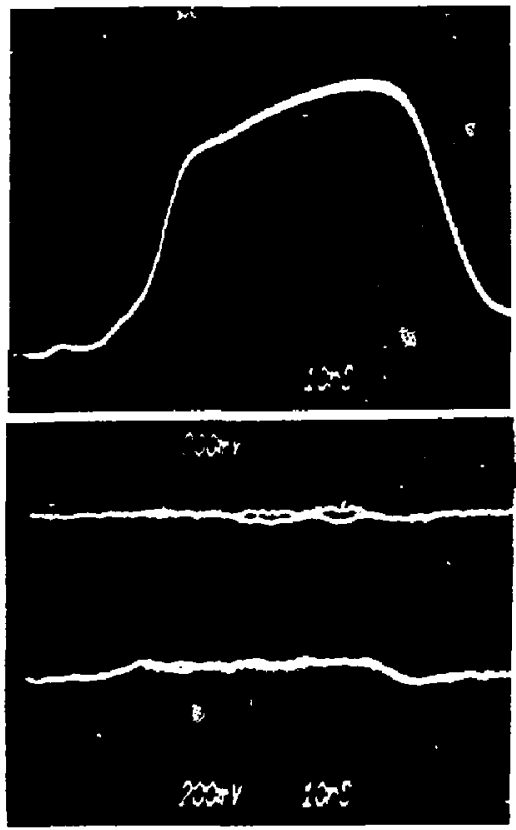

After $10 \mathrm{meV}$ (40 accelerator cores)

Same scales
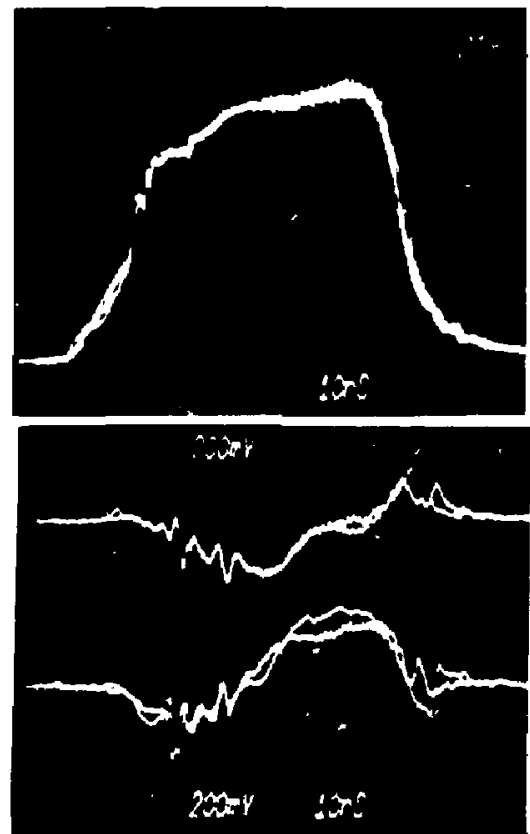
Fig. 9.

All traces are beam current at the $10 \mathrm{MeV}$ location:

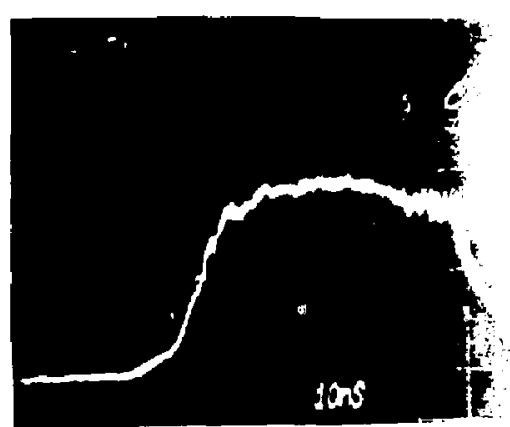

Bucking coil on

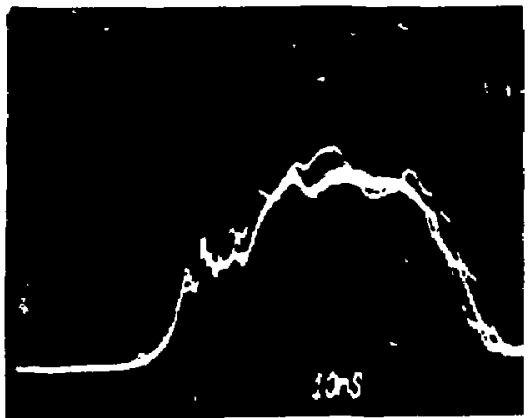

Bucking coil off

\section{Scale $1 \mathrm{kA} / \mathrm{div}$ $10 \mathrm{~ns} / \mathrm{div}$}

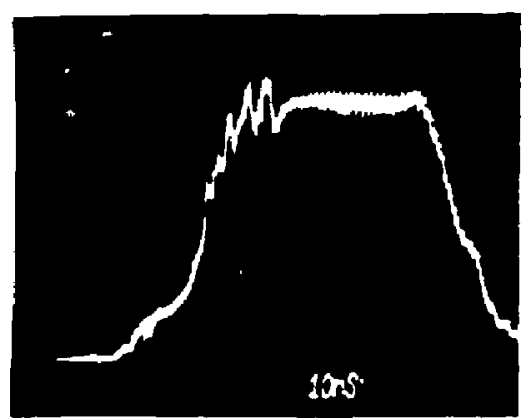

Ignitor timing $80 \mathrm{~ns}$ before grid

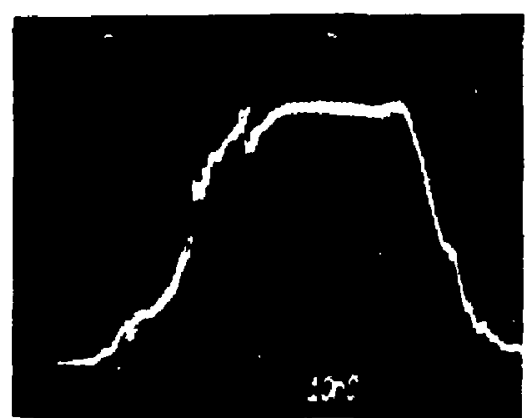

Ignitor timing 60 ns before grid

Scale $2 \mathrm{kA} / \mathrm{div}$ $10 \mathrm{~ns} / \mathrm{div}$ 

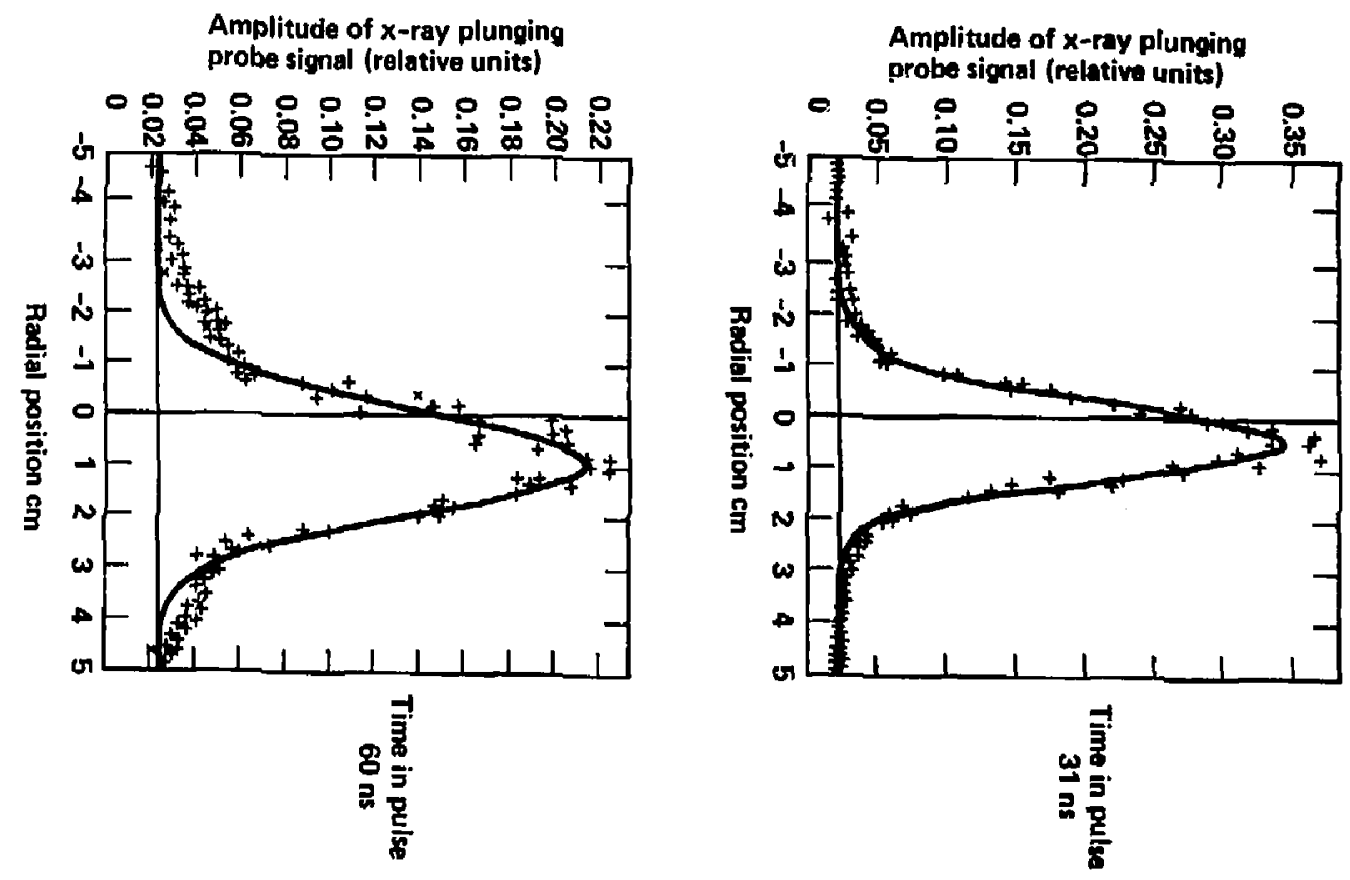
Baem characteristics at

injector output
Bewn chareteristic at

colilimatar output
Bem charecteristics at $20.0 \mathrm{MuV}$

(tranguon through 80 molenter cores)

Fig. I1.

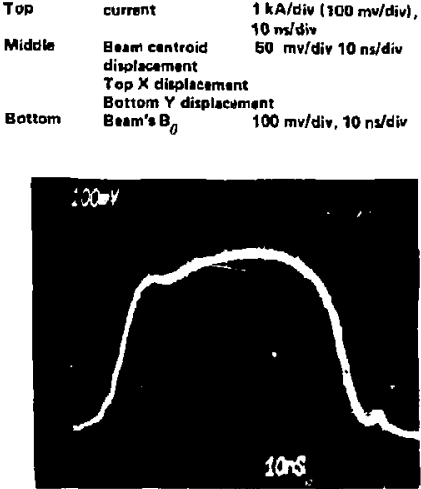

Stiy
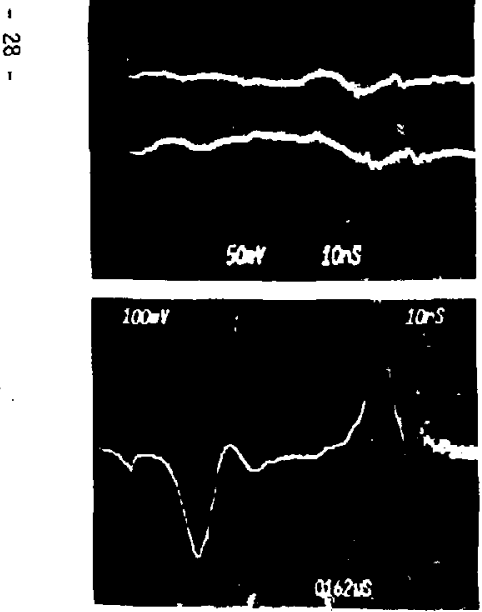

Top

Middle

$0.5 \mathrm{kA} / \mathrm{div}$ (50 mv/div), Top

10 neldiv

Beem eantraid $\quad 50 \mathrm{mv} / \mathrm{div} 20 \mathrm{n} / \mathrm{din}$

Top $X$ dinplecement

Acttom $Y$ dienlacemens

toum B, $200 \mathrm{mw} / \mathrm{div}, 10 \mathrm{~ns} / \mathrm{div}$
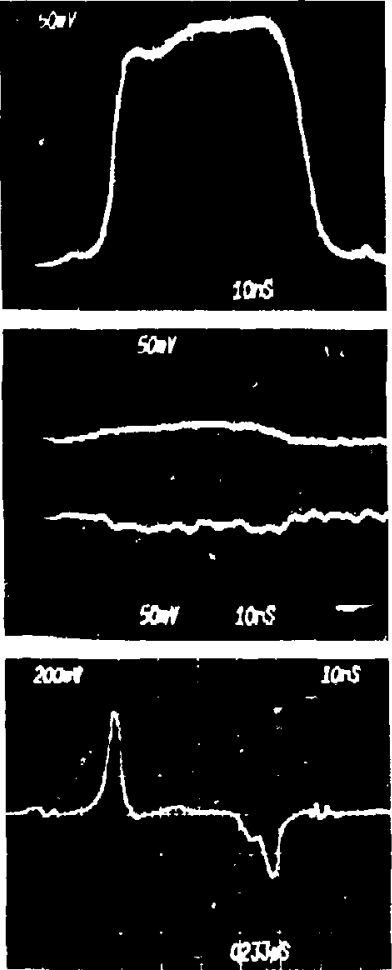

$0.5 \mathrm{kA}$ dir (50 m/dir)

current

$50 \mathrm{mu} / \mathrm{div}$,

Been ceneroid

Top $x$ dioplecement

Gottom $Y$ diphomt

Bottom Eemen $B_{0} 80 \mathrm{mw} / \mathrm{div}, 10 \mathrm{nd} / \mathrm{div}$
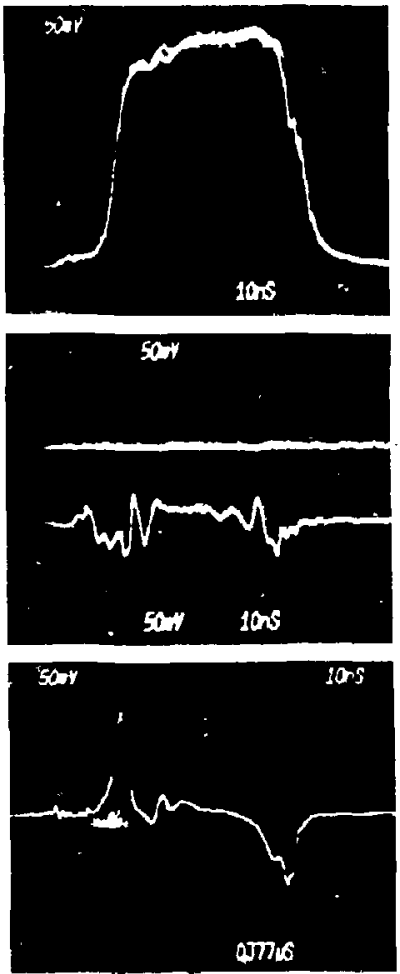
Fig. 12 .

Beam characteristics at $\sim 45 \mathrm{MeV}$ (after 180 accelerator cores) but with $6 \mathrm{~cm}$ dia $(1.06 \mathrm{~cm}$ long) collimator between injector and accelerator

Top: current $0.5 \mathrm{kA} / \mathrm{div}(50 \mathrm{mv} / \mathrm{div} 10 \mathrm{~ns} / \mathrm{div}$ ) Middle:

Beam centroid displacement

(500 mv/div) $10 \mathrm{~ns} / \mathrm{div}$

Top $X$ displacement

Bottoin $Y$ displacement

Bottom: beam's $B_{\theta} 200 \mathrm{mv} / \mathrm{div}$
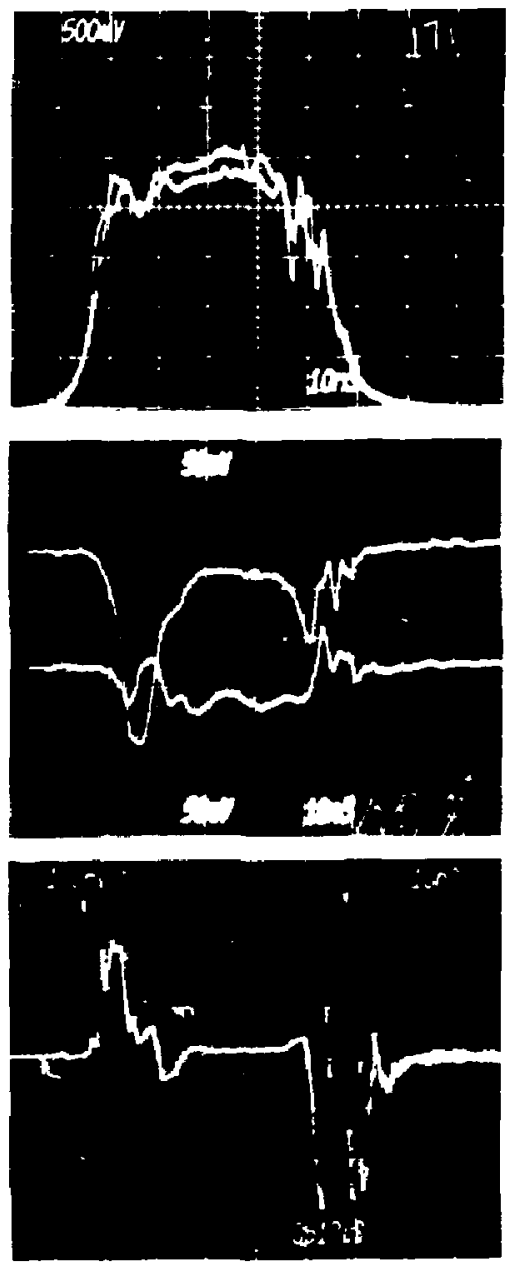

Beam characteristics at $\sim 45 \mathrm{MeV}$ !after 180 accelerator cores) but with $4 \mathrm{~cm}$ dia $(1.06 \mathrm{~cm}$ long) collimator between injector and accelerator

Top: current $05 \mathrm{kA} / \mathrm{div}$ (50 mv/div ns/div)

Middle:

Beam centroid displacement

50 mv/div 10 ns/div

Top $X$ displacement

Bottom $Y$ displacement

Bottom: beam's B $100 \mathrm{mv} / \mathrm{div} 10 \mathrm{~ns} / \mathrm{div}$
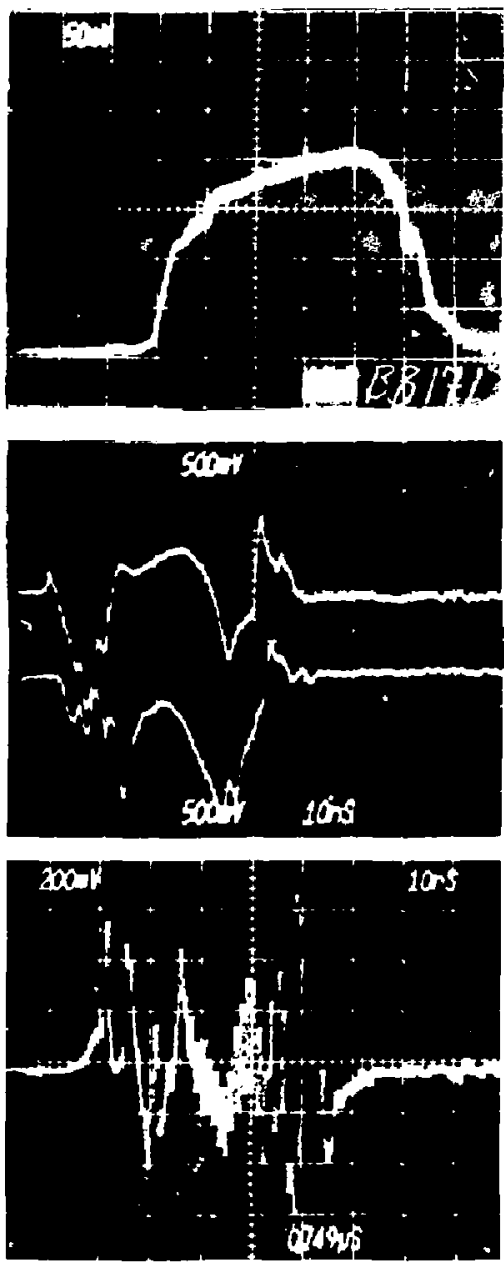
Fig. 13.

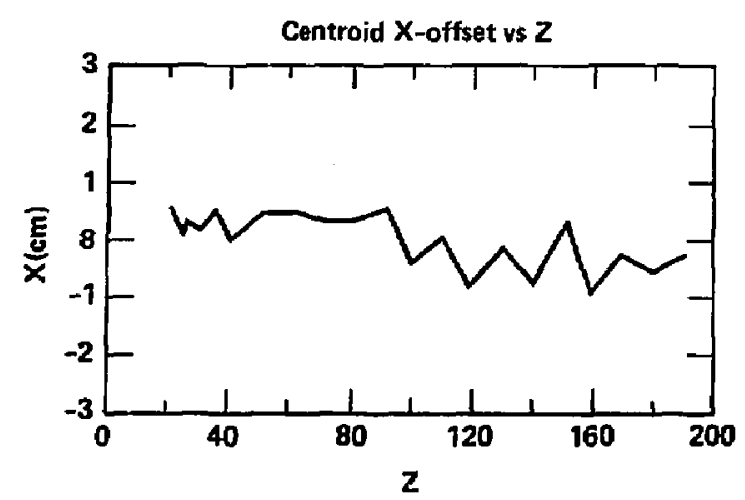

Beambug number

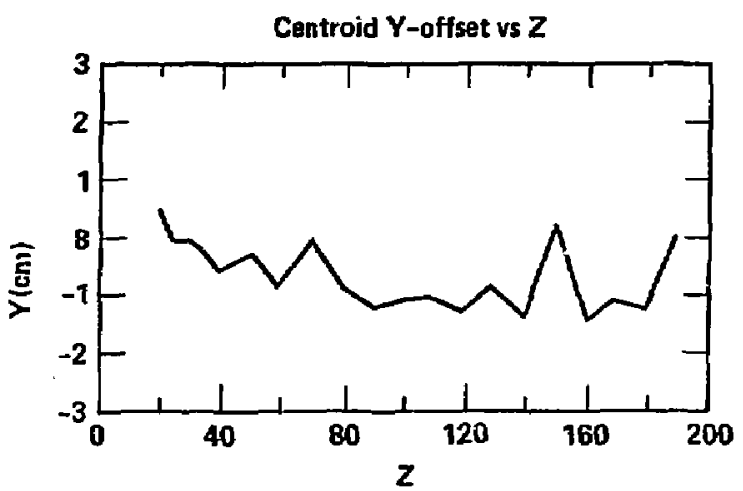

Beambug number

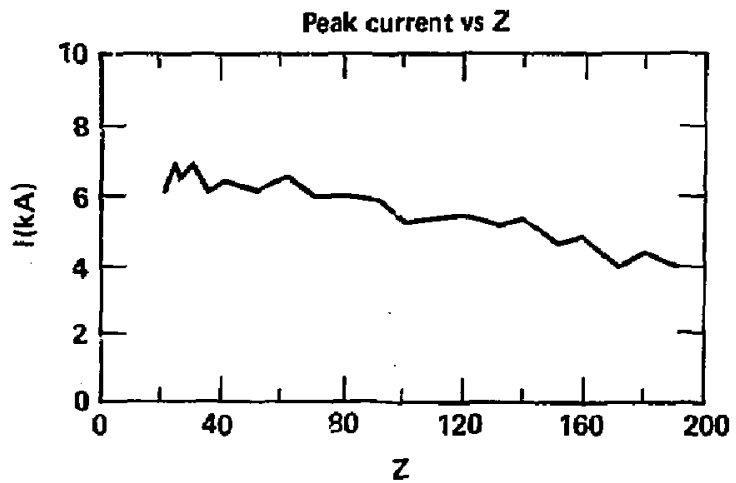

Beambug number 